\title{
Role of prostate magnetic resonance imaging in active surveillance
}

\author{
Xiaosong Meng ${ }^{1}$, Andrew B. Rosenkrantz ${ }^{2}$, Samir S. Taneja ${ }^{1,2}$ \\ ${ }^{1}$ Department of Urology, ${ }^{2}$ Department of Radiology, NYU Langone Medical Center, New York, NY 10016, USA \\ Contributions: (I) Conception and design: All authors; (II) Administrative support: SS Taneja; (III) Provision of study materials or patients: All authors; \\ (IV) Collection and assembly of data: X Meng; (V) Data analysis and interpretation: All authors; (VI) Manuscript writing: All authors; (VII) Final \\ approval of manuscript: All authors. \\ Correspondence to: Xiaosong Meng. 150 E 32 $2^{\text {nd }}$ St, New York, NY 10016, USA. Email: Xiaosong.meng@nyumc.org.
}

\begin{abstract}
Active surveillance (AS) has emerged as a beneficial strategy for management of low risk prostate cancer (PCa) and prevention of overtreatment of indolent disease. However, selection of patients for AS using traditional 12-core transrectal prostate biopsy is prone to sampling error and presents a challenge for accurate risk stratification. In fact, around a third of men are upgraded on repeat biopsy which disqualifies them as appropriate AS candidates. This uncertainty affects adoption of AS among patients and physicians, leading to current AS protocols involving repetitive prostate biopsies and unclear triggers for progression to definitive treatment. Prostate magnetic resonance imaging (MRI) has the potential to overcome some of these limitations through localization of significant tumors in the prostate. In conjunction with MRI-targeted prostate biopsy, improved sampling and detection of clinically significant PCa can help streamline the process of selecting suitable men for AS and early exclusion of men who require definitive treatment. MRI can also help minimize the invasive nature of monitoring for disease progression while on AS. Men with stable MRI findings have high negative predictive value for Gleason upgrade on subsequently biopsy, suggesting that men may potentially be monitored by serial MRI examinations with biopsy reserved for significant changes on imaging. Targeted biopsy on AS also allows for specific sampling of concerning lesions, although further data is necessary to evaluate the relative contribution of systematic and targeted biopsy in detecting the $25-30 \%$ of men who progress on AS. Further research is also warranted to better understand the nature of clinically significant cancers that are missed on MRI and why certain men have progression of disease that is not visible on prostate MRI. Consensus is also needed over what constitutes progression on MRI, when prostate biopsy can be safely avoided, and how to best utilize this additional information in current AS protocols. Despite these challenges, prostate MRI, either alone or in conjunction with MRI-targeted prostate biopsy, has the potential to significantly improve our current AS paradigm and rates of AS adoption among patients moving forward.
\end{abstract}

Keywords: Prostate cancer (PCa); prostate magnetic resonance imaging (prostate MRI); active surveillance (AS)

Submitted Feb 17, 2017. Accepted for publication Feb 23, 2017.

doi: $10.21037 /$ tau. 2017.05 .05

View this article at: http://dx.doi.org/10.21037/tau.2017.05.05

\section{Introduction}

Active surveillance (AS) for prostate cancer (PCa) has been historically underutilized but has grown in popularity recently among patients and treating physicians as a way to manage the disease without the morbidity of definitive treatment (1). The approach is supported by studies demonstrating that AS is a durable option with good long term cancer control in properly selected patient (2-4). Recently published updates from large Swedish, US, and Canadian cohorts demonstrate adherence to AS in $60 \%$ and $64 \%$ of men at 5 years $(2,5)$ and $55 \%$ of men at 15 years (3), respectively. Studies have also shown remarkably high $\mathrm{PCa}-$ specific survival rates for men on AS, ranging from $94.3 \%$ in the University of Toronto cohort (3) to $99.9 \%$ in the Johns Hopkins Hospital cohort (4) at 15 years. 
Despite good outcomes with AS, contemporary management with AS is limited by lack of standardized surveillance protocols and poorly defined criteria for proceeding to intervention. The necessity for repeated prostate biopsy among men on AS further exposes them to the morbidity associated with transrectal prostate biopsy, including acute urinary retention, urinary tract infection, bacteremia, and sepsis. A recent study in men on AS found that their odds of infection increased 1.3 times for every prior biopsy (6), which is especially concerning given the rising rates of multi-drug resistant bacteria and economic burden of hospitalization from severe infections after transrectal biopsy (7).

Successful management with AS also requires proper candidate selection. A large barrier to successful enrollment and acceptance of AS among patients and their treating physicians is the concern of missing clinically significant cancer and thus losing the opportunity for curative treatment. Traditional enrollment for AS is based upon transrectal ultrasound (TRUS)-guided systematic prostate biopsy (SB), which only samples a small proportion of the gland and is prone to sampling error (8). This leads to an underestimation of the aggressiveness and extent of disease, with around a third to almost half of men found to have Gleason score (GS) upgrade on radical prostatectomy $(9,10)$. The poor accuracy of SB in characterizing disease aggressiveness has resulted in the use of repetitive biopsy in men with low risk disease wishing to pursue or remain on AS. In recent years, magnetic resonance imaging (MRI) of the prostate has shown high performance in localizing significant tumors in the prostate $(11,12)$. MRI assists identification of tumors in areas not well sampled by SB, such as the anterior and apical aspects of the prostate (13).

Multiple studies have demonstrated improved detection of high-grade cancers using MRI/US fusion-targeted prostate biopsy compared with standard systematic biopsy alone (14-16). Given published upgrade rates among contemporary AS cohorts ranging from 24-36\% $(2,4,17)$, MRI-targeted biopsy, in conjunction with SB, may allow for earlier identification of men who are poor surveillance candidates to undergo definitive treatment, sparing them the morbidity of multiple rounds of intervening prostate biopsies prior to treatment. Monitoring of all remaining men without clinically significant disease on AS may also be more cost-effective and yield better quality-adjusted life years compared to current management with TRUSguided biopsy and serial PSA alone, as suggested by an recent Australian modeling study (18). In this article, we review the contemporary experience with prostate MRI and MRI-targeted prostate biopsy in the management of men considering AS or in an AS protocol and discuss the potential role for targeted prostate biopsy to improve current AS paradigms in candidate selection and monitoring on AS.

\section{Candidate selection for AS}

The initial challenge for selecting candidates for AS is identifying the population of men with low risk PCa that is unlikely to affective their longevity, can be safely observed, and who are interested in deferring treatment. However, while variation in the definition of low risk or clinically insignificant disease among multiple centers has led to studies with differing AS enrollment criteria, many recent studies have used the following criteria: GS $\leq 3+3$, PSA $\leq 10 \mathrm{ng} / \mathrm{mL}$, stage $\leq \mathrm{T} 2$ and either $\leq 2$ positive cores or $\leq 50 \%$ per core $(1,4,6,13,19)$. Multiple PCa risk calculators including D'Amico, Epstein and Cancer of the Prostate Risk assessment (CAPRA) incorporate these clinicalpathologic criteria to help assess eligibility for AS (20). Men who do not fulfill these criteria are deemed to have clinically significant disease and would be inappropriate for AS. In addition, given the inherent sampling error and high rate of underestimation of the aggressiveness and extent of disease with SB (8-10), men enrolling in current AS protocols typically undergo repeat prostate biopsy within one year after initial diagnosis.

Multiple studies have examined the use of MRI of the prostate prior to repeat biopsy in men considering AS. When compared to prostatectomy specimens as the reference standard, Turkbey et al. demonstrated that MRI is just as sensitive as D'Amico, Epstein, and CAPRA for determining PCa risk and may even provide additional advantages in selecting men for AS (21). The presence of visible lesions on MRI can be a negative prognostic factor for low risk disease. In a retrospective analysis of men with PCa diagnosed with SB who met the Prostate Cancer Research International: Active Surveillance (PRIAS) criteria, men with visible lesions on pre-operative MRI prior to radical prostatectomy were more likely to have their cancer status upgraded (50\% vs. $14 \%)$ and be diagnosed with unfavorable disease (52\% vs. 14\%) compared with men with no visible cancer on imaging (22). In a prospective study of patients on AS, Henderson et al. found that the apparent diffusion coefficient (ADC) obtained from the pre-enrollment MRI was a significant predictor of time to 
adverse histology on biopsy or time to radical treatment. Men with baseline ADC values below the median (972 $\mathrm{mm}^{2} / \mathrm{s}$ in the study) progressed on average seven years earlier than men with ADC values above the median (23). MRI may also better identify other exclusions for AS compared to SB such as extra-prostatic extension, seminal vesicle involvement, or lymphadenopathy $(21,24,25)$. While the overall cohort was small, Margel et al. reported in a prospective study of 60 men that $32 \%$ were reclassified following MRI and repeat conventional biopsy. Of these men, only $8.6 \%$ with a normal MRI were reclassified, compared to $77 \%$ of men with a $>1 \mathrm{~cm}$ lesion on MRI (26). Studies have also demonstrate good specificity of MRI for evaluation of extra-prostatic extension, supporting the exclusion of men from AS based on the presence of EPE on MRI $(27,28)$.

The association between level of suspicion on pre-biopsy MRI and likelihood of detection of significant PCa has been demonstrated in multiple studies $(15,29,30)$. In addition, studies have indicated that a normal MRI has a high negative predictive valve for clinically significant $\mathrm{PCa}$ on biopsy (16,31-33). Likewise, studies have demonstrated the association between MRI suspicion scores and the rate of upgrade on repeat biopsy in men considering AS (34-36). Vargas et al. reported on 388 consecutive men with clinically low risk $\mathrm{PCa}$ on initial biopsy who underwent pre-biopsy MRI prior to risk stratification biopsy and found that $20 \%$ men were upgraded on repeat biopsy (36). In that study, up to $52 \%$ of men had MRI lesions; an MRI suspicion score of 2 or less (based on a 5 point Likert scale) demonstrated high negative predictive value of $0.96-1.0$ for upgrading on confirmatory biopsy. In contrast, a suspicion score of 5 had a sensitivity of $0.87-0.98$ for upgrade on confirmatory biopsy (36).

Targeted prostate biopsy has the potential to improve risk assessment through improved sampling of suspicious lesions on MRI in men considering AS. One method of targeted biopsy utilizes in-bore MRI guided biopsy which allows for real-time feedback of needle placement and fewer sampled cores, but does not routinely sample the remaining gland (37). Using in bore magnetic resonance-guided biopsy (MRGB) of the prostate in 54 consecutive patients with low-risk PCa undergoing inclusion biopsy for AS, Somford et al. detected PCa in $55 \%$ on repeat biopsy with GS upgrade in $11 \%$ of these men. MRI lesions leading to GS upgrade had significantly lower mean ADC compared to those where only low-grade PCa was detected (38). In total, $45 \%$ of men had negative targeted biopsies despite prior histologic diagnosis of $\mathrm{PCa}$ on $\mathrm{SB}$, while repeat $\mathrm{SB}$ was not performed. In contrast to the low rates of GS upgrade in the Somford study, Felker et al. detected PCa in $69 \%$ of the 80 men in their AS sub-cohort, with $49 \%$ GS upgrade from prior SB (39). Detection of clinically significant disease increased with higher PI-RADS categories, from only $10 \%$ in PI-RADS 3 to 84\% in PI-RADS 5 lesions (39).

MRI-US fusion targeted biopsy (MRF-TB) entails targeting of MRI lesions using a variety of commercial platforms that perform co-registration of the US image and MRI during prostate biopsy (37). These systems facilitate obtaining targeted samples from MRI visible lesions as well as readily sample the remaining prostate through traditional 12 core systematic biopsies. Prior studies in large cohorts of patients have demonstrated increased detection of highgrade cancers and decreased detection of low-grade cancers with MRF-TB compared to SB, although many of these studies grouped men with mixed biopsy indications (no prior biopsy, prior negative biopsy and prior cancer on SB) for analysis $(14-16,40,41)$. Some studies have focused exclusively on men under consideration for AS. In a cohort of 281 men eligible for AS based on prior SB, Ouzzane et al. found lesions in $58 \%$ of men on MRI, with fusion targeted biopsy reclassifying $10 \%$ of men as ineligible for AS based on GS upgrade or large tumor volume (34). Of these reclassifications, $75 \%$ were in men with MRI suspicion score 4 or 5 and $54 \%$ were in the anterior prostate. Figure 1 demonstrates an example of anterior disease missed on SB that was detected with MRI and targeted biopsy from our cohort. Stamatakis et al. found that combined systematic and targeted biopsy lead to reclassification in $29 \%$ of their cohort of men eligible for AS, of which $60 \%$ of reclassifications were due to GS upgrade and the remaining due to increases in percent core length or number of positive cores (42). Hu et al. found similar reclassification rates of $36 \%$ on confirmatory systematic and targeted biopsy in 113 men enrolling in AS, with $63 \%$ of men reclassified due to GS upgrade to GS 7 or greater and $37 \%$ due to higher volume of GS 6 disease (35). Men with MRI scores of 4 or 5 were reclassified three times as often as men with scores of 2 or 3 (35), again demonstrating the consistent association between high MRI suspicion scores and detection of significant PCa. While systematic and targeted biopsy results were concordant in $50 \%$ of cases, systematic biopsy did detect significant cancer in $11 \%$ of men in who targeted biopsy did not find cancer (35).

In contrast to repeat prostate biopsy in men with prior negative SB where only targeted biopsy may be considered 

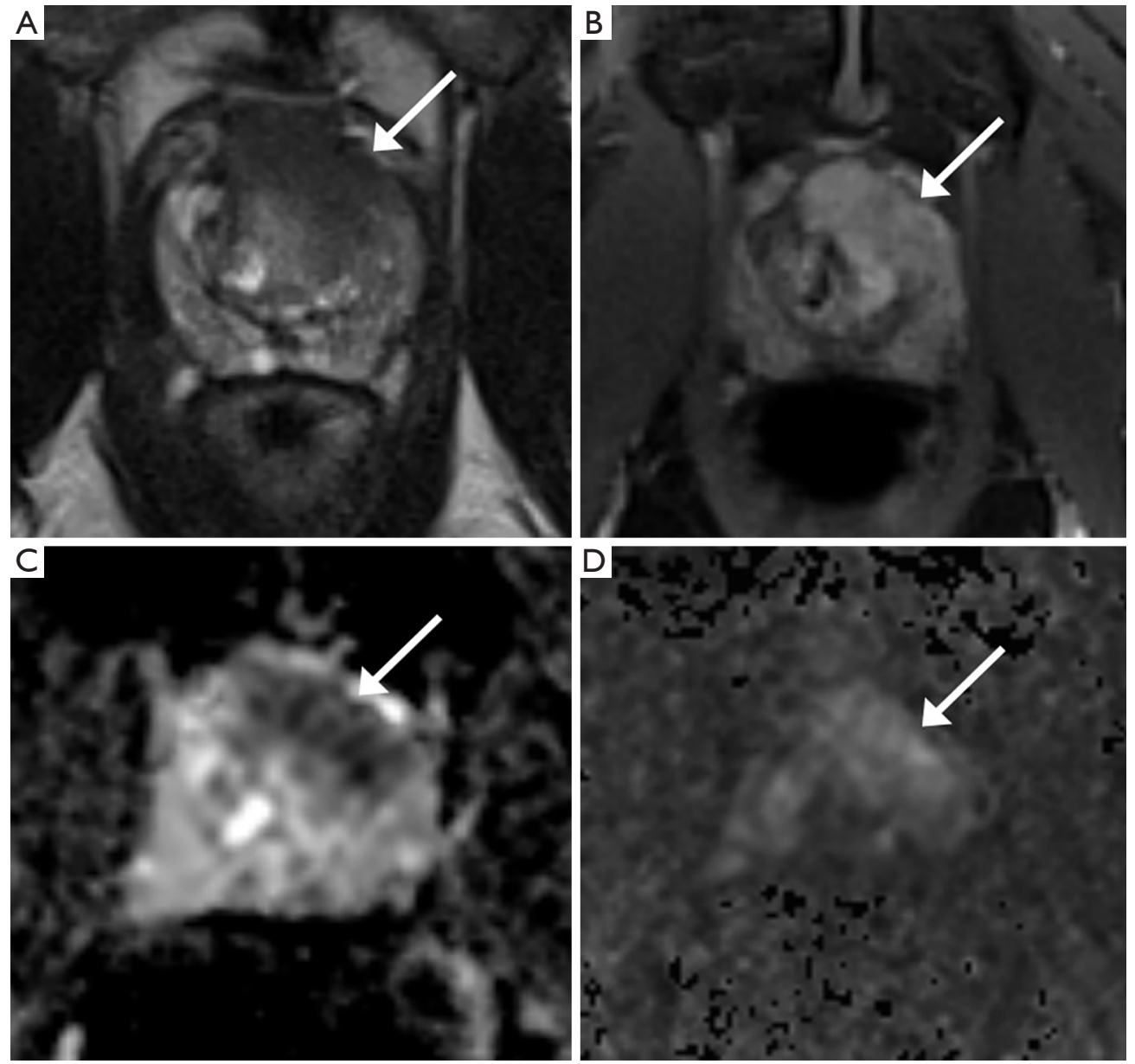

Figure 1 Value of MRI for candidate selection for active surveillance. In this example, a 60 -year-old male with rising PSA from 8.4 to 9.9 ng/mL over a 2-year period with two prior transrectal-guided biopsy demonstrating Gleason 3+3 in 1/12 cores each time in the left lateral apex. The patient was interested in pursuing active surveillance and underwent MRI for risk stratification. (A) Axial T2-weighted image shows anterior mid gland to apex lesion involving both the peripheral and transition zones, with extension of tumor beyond the prostate anteriorly; (B) post-contrast image shows focal early enhancement with edges matching lesion on other sequences; (C,D) lesion demonstrates markedly decreased apparent diffusion coefficient value $(\mathrm{C})$ and marked increased signal on ultra high b-value image (D). Lesion measures 26 mm $\times 13$ mm. Lesion was categorized as PI-RADS 5 (clinically significant cancer highly likely). MRI-US fusion-guided targeted biopsy demonstrated Gleason 4+5 and 5+4 cancer in 4/4 cores. Patient subsequently underwent robotic radical prostatectomy demonstrating T3aN0, Gleason $5+4$ prostate cancer with positive surgical margins at the bladder neck and underwent adjuvant radiation for his high risk pathology with appropriate PSA nadir following radiation. MRI, magnetic resonance imaging.

given the low detection rate of significant cancer from concordant systematic biopsy $(43,44)$, targeted biopsy alone in men under consideration for AS appears to miss significant cancer. The difference in reclassification rates between the Ouzzane study and the latter two studies is likely in part related to whether reclassification is determined by targeted biopsy alone versus combined systematic and targeted biopsy. While targeted biopsy clearly identifies significant cancer that is missed by concurrent systematic biopsy in men under consideration for AS, there is persistent concern regarding the occasional missed significant cancer on TB alone (45). Thus, performing concurrent systematic and targeted biopsy has been advised for optimal risk stratification in this population $(46,47)$.

\section{Disease progression on AS}

AS allows for monitoring of favorable cancers to delay or 

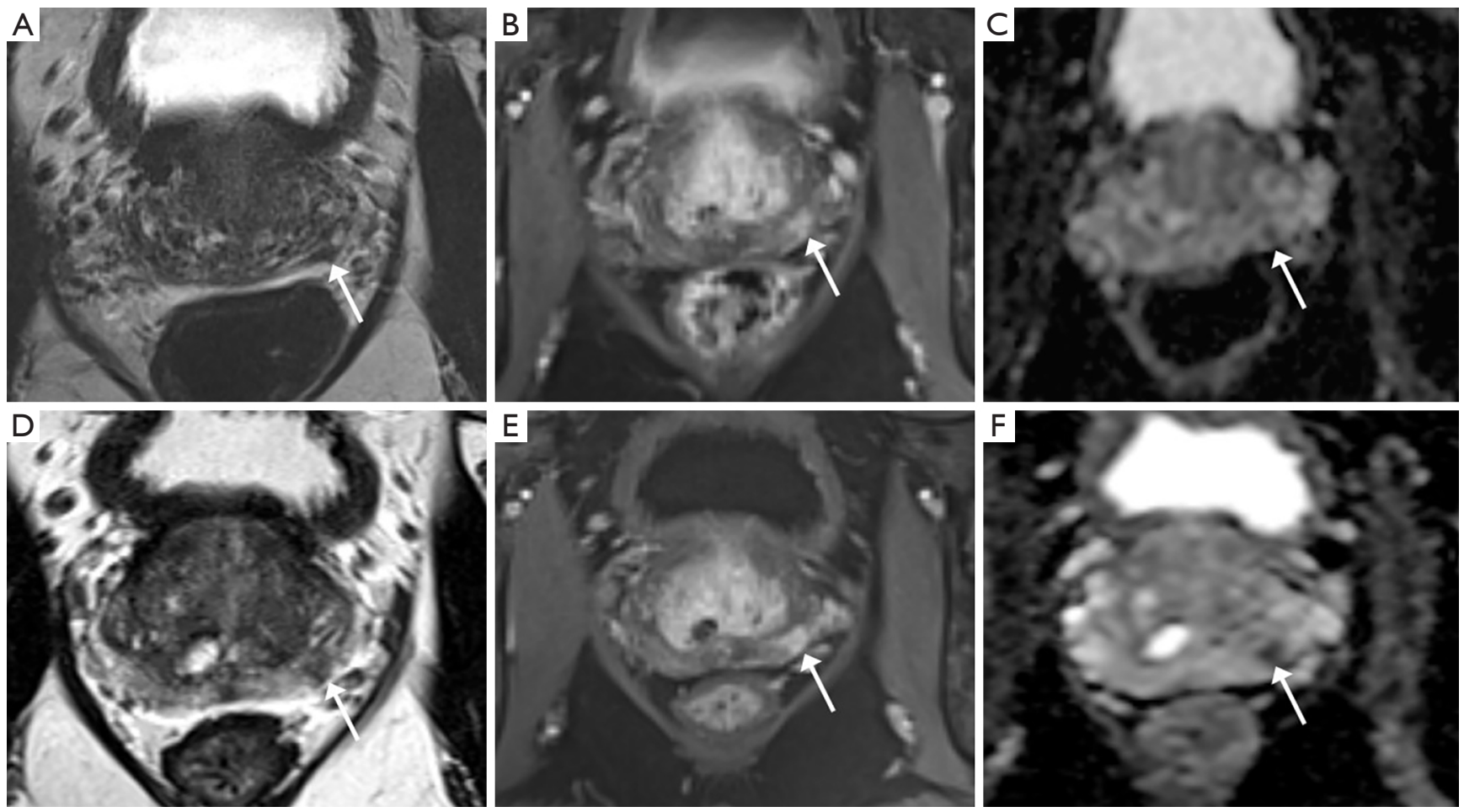

Figure 2 Detection of disease progression on active surveillance with MRI. In this example, a 65 -year-old male with PSA of $3.56 \mathrm{ng} / \mathrm{mL}$ and known Gleason 3+3 prostate cancer in 2/12 cores with increasing lesion size on follow up prostate MRI. (A-C) Baseline MRI demonstrating left posteromedial base peripheral zone lesion on axial T2-weighted image (A), early post-contrast image (B) and apparent diffusion coefficient map (C) measuring $3 \mathrm{~mm} \times 5 \mathrm{~mm}$ in size; (D-F) follow-up MRI obtained 1 year later demonstrates increase in size of lesion to $8 \mathrm{~mm} \times 8 \mathrm{~mm}$ in size, as demonstrated on axial T2-weighted image (D), early post-contrast image (E) and apparent diffusion coefficient map (F). Lesion was categorized as PI-RADS 4 (clinically significant cancer likely). MRI-US fusion-guided targeted biopsy demonstrated Gleason 3+4 and 4+3 cancer in 3/4 cores with Gleason 3+3 and 3+4 in 2/12 cores on systematic biopsy. Patient subsequently underwent robotic radical prostatectomy demonstrating $\mathrm{T} 2 \mathrm{cN} 0$, Gleason $3+4$ prostate cancer with negative surgical margins. MRI, magnetic resonance imaging.

avoid treatment unless necessary to avoid overtreatment of insignificant cancers. With this approach, accurate assessment of burden of disease is essential to prevent patients from missing their window for treatment with curative intent upon detection of higher risk or clinically significant disease. While there is no consensus for disease progression, triggers such as PSA kinetics, upgrading in GS score with pattern 4 or 5 , and increased volume of disease are generally used as criteria (48). In this role, MRI functions as an adjunct to the clinical exam, serum PSA, and serial prostate biopsies that form the traditional basis for monitoring, as well as to some of the newer urinary markers, genomic tests, and other PSA-based measurements (48) (Figure 2). In large prospective AS cohorts such as the Sunnybrook cohort, MRI has been employed as an adjunctive test in patients with adverse PSA kinetics (3). In a prospective study utilizing a subset of the Sunnybrook cohort, 72 men with a median time of 38 months on AS were referred for biopsy due to rising PSA and underwent pre-biopsy MRI followed by targeted and systematic 12-core biopsy demonstrating a reclassification rate of $43 \%$ for clinically significant cancer (49). Of these reclassifications, $26 \%$ were due to upgrade to GS 7 while 17 were was due to GS 6 with $>50 \%$ involvement in any core. Overall, targeted biopsy detected more cancers alone than systematic biopsy, and in the cases where two biopsy methods had concordant results, targeted biopsy had significantly higher maximum cancer-core lengths compared to systematic (49). As was the case with confirmatory biopsies, for men on AS, the MRI score correlated with cancer detection rates, with PPV of $49 \%$ for GS 7 cancer and 78\% for GS 6 with any core with $>50 \%$ involvement for men with MRI score $\geq 4$ and a NPV of $97 \%$ for GS $\geq 7$ cancer with a MRI score $\leq 3$ (49).

Other recent studies have examined the reclassification 
rates of men undergoing MRI and targeted prostate biopsy as part of the routine monitoring process while enrolled in AS protocols. Of the 130/253 men in the Ouzzane cohort who elected for AS after confirmatory targeted biopsy, repeat biopsy and MRI at 1 year follow-up led to reclassification in 29\% (34). However, given the low reclassification rate of only $10 \%$ on initial targeted biopsy in this cohort (34), it is likely that a subset of these upgrades on repeat biopsy may be attributed to targeting error on initial biopsy rather than progression of disease. Ma et al. recently reported on a AS cohort of 103 men with a median follow-up of 5 years and 3 prior biopsies who underwent combined SB and TB after MRI demonstrating $24 \%$ upgrade to $G S \geq 7$. Of note, SB detected a significantly larger proportion of GS $\geq 7$ compared to TB ( $20 \% v s .7 \%)$, with addition of TB to SB only leading to detection of additional $4 \%$ of $\mathrm{GS} \geq 7$ cancer (50). SB also detected $56 \%$ cancer overall and $10 \%$ GS $\geq 7$ cancer in the 127 on AS with PI-RADS 1 or 2 lesions who only underwent SB (50). Frye et al. also recently published a study examining the rates of progression of disease for 166 men on AS who were followed by targeted prostate biopsies using a fusion platform and observed progression in $30 \%$ of men over a mean follow-up of 25.5 months. In contrast to the Ma study, of the 49 men who progressed on repeat biopsy, ТВ identified significantly more men who progressed compared to $\mathrm{SB}$ ( $45 \%$ versus $31 \%$ ), with progression detected in $25 \%$ by both biopsy techniques (51).

While AS was only offered to men with small volume GS $6 \mathrm{PCa}$ in the original Epstein criteria, various programs have expanded the criteria to include men with more extensive GS 3+3 lesions or small volume GS 3+4 PCa (48). Pathologic upgrading has been observed more often in men with GS $3+4$ and intermediate risk PCa compared to men with low risk lesions in prior cohorts utilizing conventional TRUS biopsy for enrollment (48). However, as MRI and fusion targeted biopsy holds the potential to more accurately exclude men with clinically significant cancer from AS, questions remain regarding progression rates on AS in a cohort selected by fusion targeted biopsy. To help answer this question, Nassiri et al. evaluated the upgrade rate in 196 men with GS 6 and 63 men with GS 3+4 PCa who were placed on AS after diagnosis by fusion targeted biopsy. Overall, $13 \%$ of men progressed to GS $\geq 4+3 \mathrm{PCa}$. However, men with initial GS $3+4 \mathrm{PCa}$ on diagnosis were 2.8 times ( $25 \%$ vs. 9\%) more likely to progress to GS $\geq 4+3 \mathrm{PCa}$ at 3 years compared to men with only initial GS $6 \mathrm{PCa}$ (52). Other independent predictors of upgrade in the study include PSA density (PSAD) $\geq 0.15 \mathrm{ng} / \mathrm{mL} / \mathrm{cm}^{3}$ and presence of a MRI score 5 lesion (52).

Further long term outcome data is necessary to establish the role of monitoring for disease progression using serial MRI examinations instead of biopsy in men on AS. In a pilot study by Morgan et al., ADC values were found to be significantly reduced over serial MRI examinations two years apart in men who progressed to radical treatment based on PSA velocity or adverse features on biopsy compared to men who remained on AS (53). A 10\% reduction in tumor ADC values indicated progression with $93 \%$ sensitivity in the study (53). Conversely, in a cohort of 58 men on AS undergoing MRI both at baseline and prior to repeat biopsy, stable MRI findings had a negative predictive value of $80 \%$ for GS upgrade on biopsy (54). This early data suggests a potential role for non-invasive monitoring of patients with serial MRI at set intervals, with prostate biopsies only performed for changes on MRI. This practice holds the potential to significantly decrease the number of prostate biopsies performed for men on AS. Using a MRI based AS nomogram to determine whether repeat biopsy was indicated, Siddiqui et al. reports that around $47-68 \%$ of the AS cohort could potentially be spared prostate biopsy based on MRI findings (55). Further data is also needed regarding rates of upgrade or reclassification for men with stable MRI findings but changes in PSA or clinical exam over time to help inform whether prostate biopsy is necessary in this setting.

\section{Conclusions}

Prostate MRI, either alone or in conjunction with MRItargeted prostate biopsy, has the potential to significantly improve our current AS paradigm, both in early exclusion of men unsuitable for AS and improved monitoring of men on AS. Improved risk stratification using MRI and targeted prostate biopsy would help allay patient and physician concerns over missed occult clinically significant cancer. Improved confidence in the candidate selection process for AS will only increase its utilization moving forward. While recent literature supports the use of MRI and targeted prostate biopsy in men with known $\mathrm{PCa}$, there are important questions remaining to be answered. For example, reclassification rates on repeat biopsy are heterogeneous across studies, and data are conflicting regarding the relative performance of systematic versus targeted biopsy in the AS setting. Further research is warranted to better understand the nature of clinically significant cancers that are missed 
on MRI, and why certain men have progression of disease that is not visible on prostate MRI. Consensus is also needed over what constitutes progression on MRI, when prostate biopsy can be safely avoided, and how to best utilize this additional information in current AS protocols. Such insights will expand upon recent advancements in the field to further optimize the clinical management of patients on AS.

\section{Acknowledgements}

None.

\section{Footnote}

Conflicts of Interest: The authors have no conflicts of interest to declare.

\section{References}

1. Cooperberg MR, Carroll PR. Trends in Management for Patients With Localized Prostate Cancer, 1990-2013. JAMA 2015;314:80-2.

2. Loeb S, Folkvaljon Y, Makarov DV, et al. Five-year nationwide follow-up study of active surveillance for prostate cancer. Eur Urol 2015;67:233-8.

3. Klotz L, Vesprini D, Sethukavalan PK, et al. Long-term follow-up of a large active surveillance cohort of patients with prostate cancer. J Clin Oncol 2015;33:272-7.

4. Tosoian JJ, Mamawala M, Epstein JI, et al. Intermediate and longer-term outcomes from a prospective activesurveillance program for favorable-risk prostate cancer. J Clin Oncol 2015;33:3379-85.

5. Welty CJ, Cowan JE, Nguyen H, et al. Extended followup and risk factors for disease reclassification in a large active surveillance cohort for localized prostate cancer. J Urol 2015;193:807-11.

6. Ehdaie B, Vertosick E, Spaliviero M, et al. The impact of repeat biopsies on infectious complications in men with prostate cancer on active surveillance. J Urol 2014;191:660-4.

7. Zowawi HM, Harris PN, Roberts MJ, et al. The emerging threat of multidrug-resistant Gram-negative bacteria in urology. Nat Rev Urol 2015;12:570-84.

8. Bjurlin MA, Meng X, Le Nobin J, et al. Optimization of prostate biopsy: The role of magnetic resonance imaging targeted biopsy in detection, localization and risk assessment. J Urol 2014;192:648-58.

9. Kvåle R, Møller B, Wahlqvist R, et al. Concordance between Gleason scores of needle biopsies and radical prostatectomy specimens: A population-based study. BJU Int 2009;103:1647-54.

10. Mufarrij P, Sankin A, Godoy G, et al. Pathologic outcomes of candidates for active surveillance undergoing radical prostatectomy. Urology 2010;76:689-92.

11. Rosenkrantz AB, Lim RP, Haghighi M, et al. Comparison of interreader reproducibility of the prostate imaging reporting and data system and likert scales for evaluation of multiparametric prostate MRI. AJR Am J Roentgenol 2013;201:W612-8.

12. Turkbey B, Mani H, Shah V, et al. Multiparametric 3T prostate magnetic resonance imaging to detect cancer: Histopathological correlation using prostatectomy specimens processed in customized magnetic resonance imaging based molds. J Urol 2011;186:1818-24.

13. Barrett T, Haider MA. The Emerging Role of MRI in Prostate Cancer Active Surveillance and Ongoing Challenges. AJR Am J Roentgenol 2017;208:131-9.

14. Siddiqui MM, Rais-Bahrami S, Turkbey B, et al. Comparison of MR/Ultrasound Fusion-Guided Biopsy With Ultrasound-Guided Biopsy for the Diagnosis of Prostate Cancer. JAMA 2015;313:390.

15. Meng X, Rosenkrantz AB, Mendhiratta N, et al. Relationship Between Prebiopsy Multiparametric Magnetic Resonance Imaging (MRI), Biopsy Indication, and MRI-ultrasound Fusion-targeted Prostate Biopsy Outcomes. Eur Urol 2016;69:512-7.

16. Filson CP, Natarajan S, Margolis DJ, et al. Prostate cancer detection with magnetic resonance-ultrasound fusion biopsy: The role of systematic and targeted biopsies. Cancer 2016;122:884-92.

17. Selvadurai ED, Singhera M, Thomas K, et al. Mediumterm Outcomes of Active Surveillance for Localised Prostate Cancer. Eur Urol 2013;64:981-7.

18. Gordon LG, James R, Tuffaha HW, et al. Costeffectiveness analysis of multiparametric MRI with increased active surveillance for low-risk prostate cancer in Australia. J Magn Reson Imaging 2017;45:1304-15.

19. Bokhorst LP, Valdagni R, Rannikko A, et al. A Decade of Active Surveillance in the PRIAS Study: An Update and Evaluation of the Criteria Used to Recommend a Switch to Active Treatment. Eur Urol 2016;70:954-60.

20. Bastian PJ, Carter BH, Bjartell A, et al. Insignificant Prostate Cancer and Active Surveillance: From Definition to Clinical Implications. Eur Urol 2009;55:1321-30.

21. Turkbey B, Rastinehad AR, Linehan WM, et al. Prostate Cancer : Can Identify Patients Who Are Candidates. 
Radiology 2013;268:144-52.

22. Park BH, Jeon HG, Choo SH, et al. Role of multiparametric 3.0-Tesla magnetic resonance imaging in patients with prostate cancer eligible for active surveillance. BJU Int 2014;113:864-70.

23. Henderson DR, De Souza NM, Thomas K, et al. Nine-year Follow-up for a Study of Diffusionweighted Magnetic Resonance Imaging in a Prospective Prostate Cancer Active Surveillance Cohort. Eur Urol 2016;69:1028-33.

24. Ploussard G, Epstein JI, Montironi R, et al. The contemporary concept of significant versus insignificant prostate cancer. Eur Urol 2011;60:291-303.

25. Raskolnikov D, George AK, Rais-Bahrami S, et al. Multiparametric magnetic resonance imaging and imageguided biopsy to detect seminal vesicle invasion by prostate cancer. J Endourol 2014;28:1283-9.

26. Margel D, Yap SA, Lawrentschuk N, et al. Impact of multiparametric endorectal coil prostate magnetic resonance imaging on disease reclassification among active surveillance candidates: A prospective cohort study. J Urol 2012;187:1247-52.

27. Gupta RT, Faridi KF, Singh AA, et al. Comparing 3-T multiparametric MRI and the Partin tables to predict organ-confined prostate cancer after radical prostatectomy. Urol Oncol 2014;32:1292-9.

28. Raskolnikov D, George AK, Rais-Bahrami S, et al. The Role of Magnetic Resonance Image Guided Prostate Biopsy in Stratifying Men for Risk of Extracapsular Extension at Radical Prostatectomy. J Urol 2015;194:105-11.

29. NiMhurchu E, O'Kelly F, Murphy IG, et al. Predictive value of PI-RADS classification in MRI-directed transrectal ultrasound guided prostate biopsy. Clin Radiol 2016;71:375-80.

30. Kuru TH, Roethke MC, Rieker P, et al. Histology corespecific evaluation of the European Society of Urogenital Radiology (ESUR) standardised scoring system of multiparametric magnetic resonance imaging (mpMRI) of the prostate. BJU Int 2013;112:1080-7.

31. Itatani R, Namimoto T, Atsuji S, et al. Negative predictive value of multiparametric MRI for prostate cancer detection: Outcome of 5-year follow-up in men with negative findings on initial MRI studies. Eur J Radiol 2014;83:1740-5.

32. Pokorny MR, De Rooij M, Duncan E, et al. Prospective study of diagnostic accuracy comparing prostate cancer detection by transrectal ultrasound-guided biopsy versus magnetic resonance (MR) imaging with subsequent mr- guided biopsy in men without previous prostate biopsies. Eur Urol 2014;66:22-9.

33. Wysock JS, Mendhiratta N, Zattoni F, et al. Predictive value of negative $3 \mathrm{~T}$ multiparametric magnetic resonance imaging of the prostate on 12-core biopsy results. BJU Int 2016;118:515-20.

34. Ouzzane A, Renard-Penna R, Marliere F, et al. Magnetic Resonance Imaging Targeted Biopsy Improves Selection of Patients Considered for Active Surveillance for Clinically Low Risk Prostate Cancer Based on Systematic Biopsies. J Urol 2015;194:350-6.

35. Hu JC, Chang E, Natarajan S, et al. Targeted prostate biopsy in select men for active surveillance - Do the epstein criteria still apply? J Urol 2014;192:385-90.

36. Vargas HA, Akin O, Afaq A, et al. Magnetic resonance imaging for predicting prostate biopsy findings in patients considered for active surveillance of clinically low risk prostate cancer. J Urol 2012;188:1732-8.

37. Bjurlin MA, Mendhiratta N, Wysock JS, et al. Multiparametric MRI and targeted prostate biopsy: Improvements in cancer detection, localization, and risk assessment. Cent European J Urol 2016;69:9-18.

38. Somford DM, Hoeks CM, Hulsbergen-van de Kaa CA, et al. Evaluation of diffusion-weighted MR imaging at inclusion in an active surveillance protocol for low-risk prostate cancer. Invest Radiol 2013;48:152-7.

39. Felker ER, Lee-Felker SA, Feller J, et al. In-bore magnetic resonance-guided transrectal biopsy for the detection of clinically significant prostate cancer. Abdom Radiol (NY) 2016;41:954-62.

40. Sonn GA, Natarajan S, Margolis DJA, et al. Targeted biopsy in the detection of prostate cancer using an office based magnetic resonance ultrasound fusion device. J Urol 2013;189:86-91.

41. Kuru TH, Roethke MC, Seidenader J, et al. New Technology and Techniques Critical Evaluation of Magnetic Resonance Imaging Targeted, Transrectal Ultrasound Guided Transperineal Fusion Biopsy for Detection of Prostate Cancer. J Urol 2013;190:1380-6.

42. Stamatakis L, Siddiqui MM, Nix JW, et al. Accuracy of multiparametric magnetic resonance imaging in confirming eligibility for active surveillance for men with prostate cancer. Cancer 2013;119:3359-66.

43. Mendhiratta N, Meng X, Rosenkrantz AB, et al. Prebiopsy MRI and MRI-ultrasound Fusion-targeted Prostate Biopsy in Men with Previous Negative Biopsies: Impact on Repeat Biopsy Strategies. Urology 2015;86:1192-8.

44. Rosenkrantz AB, Verma S, Choyke P, et al. Prostate 
Magnetic Resonance Imaging and Magnetic Resonance Imaging Targeted Biopsy in Patients with a Prior Negative Biopsy: A Consensus Statement by AUA and SAR. J Urol 2016;196:1613-8.

45. Felker ER, Margolis DJ, Nassiri N, et al. Prostate cancer risk stratification with magnetic resonance imaging. Urol Oncol 2016;34:311-9.

46. Recabal P, Assel M, Sjoberg DD, et al. The Efficacy of Multiparametric Magnetic Resonance Imaging and MRI-Targeted Biopsy in Risk Classification for Patients with Prostate Cancer on Active Surveillance. J Urol 2016;196:374-81.

47. Tran GN, Leapman MS, Nguyen HG, et al. Magnetic Resonance Imaging-Ultrasound Fusion Biopsy During Prostate Cancer Active Surveillance. Eur Urol 2016. [Epub ahead of print].

48. Tosoian JJ, Carter HB, Lepor A, et al. Active surveillance for prostate cancer: current evidence and contemporary state of practice. Nat Rev Urol 2016;13:205-15.

49. Da Rosa MR, Milot L, Sugar L, et al. A prospective comparison of MRI-US fused targeted biopsy versus systematic ultrasound-guided biopsy for detecting clinically significant prostate cancer in patients on active surveillance. J Magn Reson Imaging 2015;41:220-5.

50. Ma TM, Tosoian JJ, Schaeffer EM, et al. The Role

Cite this article as: Meng X, Rosenkrantz AB, Taneja SS. Role of prostate magnetic resonance imaging in active surveillance. Transl Androl Urol 2017;6(3):444-452. doi: 10.21037/ tau.2017.05.05 of Multiparametric Magnetic Resonance Imaging/ Ultrasound Fusion Biopsy in Active Surveillance. Eur Urol 2017;71:174-80.

51. Frye TP, George AK, Kilchevsky A, et al. Magnetic Resonance Imaging-Transrectal Ultrasound Guided Fusion Biopsy to Detect Progression in Patients with Existing Lesions on Active Surveillance for Low and Intermediate Risk Prostate Cancer. J Urol 2017;197:640-6.

52. Nassiri N, Margolis DJ, Natarajan S, et al. Targeted biopsy to detect Gleason score upgrading during active surveillance for men with low- vs. intermediate-risk prostate cancer. J Urol 2017;197:632-9.

53. Morgan VA, Riches SF, Thomas K, et al. Diffusionweighted magnetic resonance imaging for monitoring prostate cancer progression in patients managed by active surveillance. Br J Radiol 2011;84:31-7.

54. Walton Diaz A, Shakir NA, George AK, et al. Use of serial multiparametric magnetic resonance imaging in the management of patients with prostate cancer on active surveillance. Urol Oncol 2015;33:202.e1-7.

55. Siddiqui MM, Truong H, Rais-Bahrami S, et al. Clinical implications of a multiparametric magnetic resonance imaging based nomogram applied to prostate cancer active surveillance. J Urol 2015;193:1943-9. 